SISSA $68 / 97 / \mathrm{EP} / \mathrm{FM}$

hep-th/9705137

\title{
On the String Interpretation of $\mathrm{M}$ (atrix) Theory
}

\author{
L. Bonora ${ }^{a, b}$, C.S. Chu ${ }^{a}$ \\ (a) International School for Advanced Studies (SISSA/ISAS), \\ Via Beirut 2, 34014 Trieste, Italy \\ (b) INFN, Sezione di Trieste \\ E-mail: bonora@sissa.it, cschu@sissa.it
}

\begin{abstract}
It has been proposed recently that, in the framework of $\mathrm{M}$ (atrix) theory, $\mathcal{N}=8$ supersymmetric $U(N)$ Yang-Mills theory in $1+1$ dimensions gives rise to type IIA long string configurations. We point out that the quantum moduli space of $\mathrm{SYM}_{1+1}$ gives rise to two quantum numbers, which fit very well into the $\mathrm{M}$ (atrix) theory. The two quantum numbers become familiar if one switches to a IIB picture, where they represent configurations of $\mathrm{D}$-strings and fundamental strings. We argue that, due to the $S L(2, \mathbf{Z})$ symmetry, of the IIB theory, such quantum numbers must represent configurations that are present also in the IIA framework.
\end{abstract}




\section{Introduction}

It has recently been proposed [1, 2, 3], in the framework of $\mathrm{M}$ (atrix) theory [4], that IIA string theory in the light cone can be identified with the two dimensional $\mathcal{N}=8$ supersymmetric $U(N)$ Yang-Mills theory $\left(\mathrm{SYM}_{1+1}\right)$ in the $N \rightarrow \infty$ limit. This IIA theory contains not only the ordinary string configurations, but also new non-trivial string configurations, the 'long strings' [5], which naturally arise from the SYM moduli space. To stress the distinction with the usual type IIA string theory, at times we refer to the latter theory as the 'enlarged IIA theory'.

In this paper we point out that the 'quantum moduli space' of a $\mathrm{SYM}_{1+1}$ theory is characterized by a couple of quantum numbers $(m, n)$ that fit very nicely in the framework of the compactified M(atrix) theory and explain not only the 'long strings' but also other topological configurations that are traditionally not present in a IIA picture. The latter become more natural if, by a T-duality operation, we switch to a IIB picture. In a IIB context the two quantum numbers correspond to configurations of D-strings and elementary strings and it is, of course, natural for all of them to feature in the theory, due to the $S L(2, \mathbf{Z})$ symmetry of the latter. Thus, to the extent that M(atrix) theory is an exact description of $M$ theory, we must supplement IIA theory not only with long strings, but also with additional topological configurations (which will be described below).

In this regard we can also add another remark. The 'long string' configurations in [1, 2, 3] were included in the spectrum as they represent the twisted sectors of the $\mathrm{SYM}_{1+1}$ orbifold moduli space, as is usual in ordinary string orbifold constructions in order to guarantee modular invariance. The results of this paper, briefly stated above, show that the role analogous to 'modular invariance' for the enlarged IIA theory is played by the $S L(2, \mathbf{Z})$ symmetry of the IIB theory.

The paper is organized as follows. In the next section we use well-known results on YangMills theories in $1+1$ dimensions, to give an explicit representation of the 'quantum moduli space' for these theories, as anticipated above. In section 3, we apply this notion to the $\mathrm{M}$ (atrix) framework. Section 4 is devoted to some comments.

\section{SYM Theory on a Cylinder}

In this section we use well-known results on Yang-Mills theory in $1+1$ dimensions, and reformulate its moduli space in a way which is suitable to the present context. Let us consider a theory, with gauge group $G=U(N)$, defined on a circle of circumference $L=2 \pi R$ and suitably rescaled so that the pure Yang-Mills part becomes

$$
S=\frac{1}{4 g_{Y M}^{2}} \int d t d \sigma \operatorname{tr} F_{\mu \nu}^{2}
$$

where $g_{Y M}$ is the gauge coupling constant. 
The theory (18) admit the boundary conditions

$$
\phi(t, \sigma+L)=U \phi(t, \sigma) U^{\dagger},
$$

where $\phi$ is any of the matrix-fields involved and $U$ is a constant matrix in $U(N)$. We first notice that if $U$ is in the center of the gauge group, then the boundary condition (2) corresponds to periodicity. Otherwise, a generic $U$ corresponds to a theory over a noncompact space, unless $U^{k} \in U(1)$ - which without loss of generality can be reduced to $U^{k}=\mathbf{1}$ - for some minimal $k$. We are not interested in the generic case, while our attention will concentrate on the latter case which corresponds to a gauge theory defined (i.e. with periodic boundary conditions) on a $k$-th covering of the circle, that is a circle of radius $k R$. We conventionally refer to this circle as a 'large circle' when $k>1$, as opposed to the original 'small circle' of radius $R$. This splits the theory into different sectors, which, from the point of view of the gauge fixing, can be treated separately. In each sector the allowed gauge transformations are the periodic ones on the corresponding large circle.

Any $U$ satisfying $U^{k}=\mathbf{1}$ for some finite $k$ belongs to some finite discrete subgroups of $U(N)$, but, in the following, we restrict ourselves to those $U$ that belong to the Weyl group $S_{N}$ (the permutation group of $N$ objects) of $U(N)$. . And to avoid any confusion we denote the elements of $S_{N}$ by $g$, instead of $U$. Each distinct sector is specified, in this case, by a conjugacy class of $S_{N}$, denoted as $[g]$. We recall that a conjugacy class $[g]$ of $S_{N}$ corresponds to a partition of $N$ into 'cycles'. It is a theorem of finite group theory that the order $k$ of $[g]$ is the least common multiple of the degrees of the cycles contained in the partition.

We recall that, in each sector, we have periodic boundary conditions over the appropriate large circle, and the gauge fixing can therefore be carried out as in [6, 7, 8]. One first solves the holonomy equation $\left(\partial_{1}-A_{1}\right) V=0$ and, by means of the solution $V$, constructs a gauge transformation that maps $A_{1}(t, \sigma)$ to a $\sigma$-independent potential; then, by means of another $\sigma$-independent gauge transformation one brings $A_{1}$ to the diagonal form. This entails also $A_{0}=0$. In summary i

$$
A_{0}=0, \quad A_{1}(t, \sigma)=\frac{1}{L} \operatorname{diag}\left(\beta_{1}(t), \beta_{2}(t), \cdots, \beta_{N}(t)\right) .
$$

In this gauge, the electric field is given by

$$
E=\frac{1}{L} \operatorname{diag}\left(\dot{\beta}_{1}(t), \dot{\beta}_{2}(t), \cdots, \dot{\beta}_{N}(t)\right)
$$

and, since the Faddeev-Popov determinant for gauge fixing (31) is trivial, the action (11) reduces to

$$
S=\int d t \mathcal{L}, \quad \mathcal{L}=\frac{1}{2 g_{Y M}^{2} L} \sum_{k=1}^{N} \dot{\beta}_{k}^{2} .
$$

*It is an open question whether $U$ 's not belonging to $S_{N}$ may generate interesting physical configurations like the 'long strings' of the following section.

$\dagger$ In fact, the gauge fixing also implies that, if $A_{0}(t, \sigma)_{i j}=\sum_{n} a_{n}(t)_{i j} e^{2 \pi i n \sigma / L}$, then $a_{n}(t)_{i j}$ can be nonvanishing in some particular cases. In any case the $a_{n}(t)_{i j}$ 's appear in the action as Lagrange multipliers that impose conditions which are irrelevant in this paper. 
It is easy to see that the gauge fixed configurations have in general a residual gauge symmetry $\mathbf{Z}^{N}$ :

$$
\beta_{k} \rightarrow \beta_{k}+2 \pi n_{k}, \quad n_{k} \in \mathbf{Z},
$$

This amounts to restricting the $\beta_{k}$ 's to be living on the torus $T^{N}$.

In addition to (6), (3) has another residual gauge symmetry. In every sector we can go back from the 'large circle' to the 'small circle' where the theory is originally formulated. In so doing we must switch on again the boundary conditions (2). It is easy to see what the allowed boundary conditions and residual gauge transformations in each sector are. The boundary condition

$$
A_{1}(t, \sigma+L)=g A_{1}(t, \sigma) g^{\dagger}
$$

together with $A_{1}$ being diagonal, implies that, since $g$ is in $S_{N}$, the only operation it performs is to permute the $\beta_{k}$ eigenvalues among themselves. In relation to the given $g$, the set of $\beta_{k}$ 's will split uniquely into cycles within each of which $g$ acts irreducibly while the cycles are not permuted. Then, as a consequence of (7) and the $\sigma$-independence of $A_{1}$, the $\beta_{k}$ 's in each cycle must all be equal.

Now, in any sector, the residual gauge symmetry is $S_{N}$, since acting with any $h \in S_{N}$ on $A_{1}$, will preserve ([) with $g$ replaced by $h g h^{\dagger}$, i.e. will preserve the sector. The above conclusion is tantamount to saying that the moduli space of the gauge degrees of freedom of the theory (18), for each sector, is the orbifold

$$
\mathbf{R}^{N} / G_{N}
$$

where $G_{N}$ is the semidirect product of $S_{N}$ and $\mathbf{Z}^{N}$.

What we had said so far accounts for the second of the two integers announced in the introduction. The first one comes from quantization. We recall that the $\beta_{k}$ 's live on $T^{N}$. Therefore the canonical momenta $\pi_{k}$ conjugate to the $\beta_{k}$ 's are quantized,

$$
\pi_{k}=\frac{1}{g_{Y M}^{2}} E_{k}, \quad \pi_{k} \in \mathbf{Z}
$$

and the energy from the pure gauge sector is

$$
\mathcal{E}=\frac{g_{Y M}^{2} L}{2} \sum_{k=1}^{N} \pi_{k}^{2} .
$$

As an aside, let us recall that this is the so-called non-compact quantization [8], as opposed to the compact one [9] in which one directly quantizes the Wilson loops. In the compact quantization the characters $\chi_{R}$ of the irreducible representations $R$ of $U(N)$ form a basis of the Hilbert space of gauge invariant functions. The corresponding energy is given by

$$
\mathcal{E}_{R}=\frac{g_{Y M}^{2} L}{2} C_{2}(R)
$$


where

$$
C_{2}(R)=\sum_{i=1}^{N} f_{i}^{2}+\sum_{i=1}^{N}(N+1-2 i) f_{i}
$$

is the quadratic Casimir for the representation $R$ specified by a Young tableau with $f_{i}$ boxes in the $i^{\text {th }}$ row. Comparing the energies in the two quantizations we see that they agree up to the second term in the RHS of (12), which is generated by the curvature of the group manifold. Therefore one can make the identification

$$
f_{i} \equiv \pi_{i}
$$

Let us return to the non-compact quantization. In the case of twisted sectors (7) for which the $\beta_{k}$ 's within the same cycle have to be identified, one must introduce some obvious modifications due to such constraints. For example, in a subsector given by a cycle of length $n$, one gets

$$
\pi_{k}=\frac{n}{g_{Y M}^{2}} E_{k}, \quad \pi_{1}=\pi_{2}=\cdots=\pi_{n} \in \mathbf{Z}
$$

and

$$
\mathcal{E}_{\text {cycle }}=\frac{g_{Y M}^{2} L}{2 n^{2}} \sum_{k=1}^{n} \pi_{k}^{2},
$$

The analysis of the moduli space, carried out in this section, is strictly valid for pure Yang-Mills. The addition of matter may modify its structure. However this will not happens for the $\mathcal{N}=8$ supersymmetric theories we will consider in the following section.

Finally, with some abuse of language, we define what we mean by 'quantum moduli space' of a $\mathrm{SYM}_{1+1}$, like the ones considered in the next section. A point in it is a collection of subsectors, each of which is characterized by two integers $(m, n)$, where $n$ denotes the degree or length of a cycle in a given partition of $N$, and $m$ represents the common eigenvalue of the $\pi_{k}$ contained in that cycle. As we will see in the next section, these subsectors admit different stringy interpretations in different theories.

\section{IIB versus IIA}

According to the original proposal of [4], $M$ theory in the infinite momentum frame is described by the $U(N)$ supersymmetric quantum mechanics $\mathrm{SYM}_{1+0}$ in the limit $N \rightarrow \infty$

$$
\begin{aligned}
L & =\frac{1}{2} \operatorname{tr}\left(\frac{1}{R_{11}} \dot{X}^{i 2}-R_{11}\left[X^{i}, X^{j}\right]^{2}-\theta^{T} \dot{\theta}-R_{11} \theta^{T} \gamma_{i}\left[X^{i}, \theta\right]\right) \\
H & =\frac{R_{11}}{2} \operatorname{tr}\left(\Pi_{i}^{2}+\left[X^{i}, X^{j}\right]^{2}+\theta^{T} \gamma_{i}\left[X^{i}, \theta\right]\right)
\end{aligned}
$$

$R_{11}$ is the radius of the original longitudinal circle on which $\mathrm{M}$ theory is compactified. For finite $N$, the $\mathrm{SYM}_{1+0}$ describes the low energy effective dynamics (at short distances and low velocities) 110 for a system of $N$ D0 branes with string coupling $g_{s}=\left(R_{11} / l_{p}\right)^{3 / 2}$ and is a 
partial description of the compactified $M$ theory, i.e. 10 dimensional IIA string theory. The total longitudinal momentum is given by $p_{11}=N / R_{11}$. The limit $N \rightarrow \infty$ takes one to the infinite momentum frame. It was argued that all relevant velocities vanish in the same limit; this together with a conjectured nonrenormalization theorem for the $v^{4}$ term in the effective action for D0 brane justifies the use of (16) as an exact nonperturbative formulation for $\mathrm{M}$ theory in the infinite momentum frame.

Toroidal compactification of (16) for finite $N$ has been carried out in details in [11] and a two dimensional SYM model was obtained. For our purpose, we repeat this procedure in the Appendix and get the following two dimensional $\mathcal{N}=8 \mathrm{SYM}_{1+1}$ on a cylinder $0 \leq \sigma \leq 2 \pi$,

$$
S=\frac{1}{2 \pi l_{s}^{2}} \int \operatorname{tr}\left(\left(D_{\mu} X^{i}\right)^{2}+\theta^{T} \not D \theta+\frac{1}{g_{Y M}^{2}} F_{\mu \nu}^{2}-g_{Y M}^{2}\left[X^{i}, X^{j}\right]^{2}+g_{Y M} \theta^{T} \gamma_{i}\left[X^{i}, \theta\right]\right) \text {. }
$$

The fields $X^{i}, i=1, \cdots, 8$ transform in the vector representation $\mathbf{8}_{\mathbf{v}}$ of $S O(8)$, while the two spinors $\theta_{L}^{\alpha}, \theta_{R}^{\dot{\alpha}}, \alpha, \dot{\alpha}=1, \cdots, 8$ transform in the representations $\mathbf{8}_{\mathbf{s}}, \mathbf{8}_{\mathbf{c}}$ and have opposite chirality. All the fields are $N \times N$ hermitian matrices. The coupling $g_{Y M}$ depends on the parameters $R_{9}$ and $R_{11}$ and this dependence can be modified by means of field redefinitions. Later, we will use this fact to study the different stringy interpretations of (17).

$\mathrm{M}$ theory compactified on a circle is supposed to give 10 dimensional nonperturbative IIA string theory. Given the BFSS formulation for M theory, one can get a nonperturbative formulation for IIA string theory by studying the compactification of (16). The prescription of sect. 9 of [4] and [1] is to take the large $N$ limit of the SYM (17) resulting from the compactification of (16). To this purpose, the authors of [3] obtained the following supersymmetric $U(N)$ Yang-Mills theory $\left(\mathrm{SYM}_{1+1}\right)$ as a nonperturbative formulation for IIA string theory,

$$
S=\frac{1}{2 \pi} \int \operatorname{tr}\left(\left(D_{\mu} X^{i}\right)^{2}+\theta^{T} \not D \theta+g_{s}^{2} F_{\mu \nu}^{2}-\frac{1}{g_{s}^{2}}\left[X^{i}, X^{j}\right]^{2}+\frac{1}{g_{s}} \theta^{T} \gamma_{i}\left[X^{i}, \theta\right]\right) .
$$

in units $l_{s}=1$. In (18), the radius $R_{11}$ is understood and $N$ is considered in the limit $N \rightarrow \infty$. The identification with type IIA string theory in [3] is made via the flip $R_{9} \leftrightarrow R_{11}$ so that the string coupling $g_{s}$ is related to $R_{9}$ by

$$
g_{s}=\left(R_{9} / l_{p}\right)^{3 / 2}=R_{9} / l_{s} .
$$

The action (18) is not the only possible representation of (17) we want to discuss. In particular, we consider the following identifications of $g_{Y M}$ admitting stringy interpretations:

$$
\begin{aligned}
& \mathcal{A}_{D}: g_{Y M}^{2}=\left(l_{p} / R_{11}\right)^{3} / l_{s}^{2}, \\
& \mathcal{A}_{F}: g_{Y M}^{2}=\left(l_{p} / R_{9}\right)^{3} / l_{s}^{2}, \\
& \mathcal{B}_{D}: g_{Y M}^{2}=\left(R_{11} / R_{9}\right) / l_{s}^{2}, \\
& \mathcal{B}_{F}: \quad g_{Y M}^{2}=\left(R_{11} / R_{9}\right)^{2} / l_{s}^{2} .
\end{aligned}
$$

In the following, however, we set $l_{s}=1$ throughout. 
We now explain what we mean by $\mathcal{A}_{D}, \mathcal{A}_{F}$ etc.. First of all, as long as $N$ is finite, we interpret (17) as representing a 9-dimensional theory. According to the current M(atrix) theory interpretation, upon taking the limit $N \rightarrow \infty$, we are supposed to recover 10 dimensional theories. We will comment on this point at the end of the paper. Throughout this section we keep $N$ large but finite.

The most obvious interpretation comes from the very construction of the D0-brane theory of BFSS: (17) represents a theory of D0-branes with string coupling $g_{s}=R_{11}$ compactified on a circle of radius $R_{9}$ in the $9^{\text {th }}$ direction. Let us call it $\mathcal{A}_{D}$. Flipping the $9^{\text {th }}$ and $11^{\text {th }}$ dimensions, we obtain a new version of the theory, which we refer to as $\mathcal{A}_{F}$. A suitable action for this theory is (18). This theory, in the $N \rightarrow \infty$ limit, has been interpreted in [3] as a theory of IIA strings, see also [1, 2]. On the other hand, starting from $\mathcal{A}_{D}$ and performing a T-duality operation in the $9^{\text {th }}$ direction we get a theory of type IIB D-strings, [4, 11]. For finite $N$, we recall that (17) was obtained by compactifying 10 dimensional D0 dynamics on a circle of radius $R_{9}$, so by $\mathrm{T}$-duality, (17) represents a collection of $N$ D-strings, with 'coordinates' $X^{i}, \theta^{i}$, stretched in the $9^{t h}$ direction [12] on a circle of radius $1 / R_{9}$, with the string coupling

$$
g_{B}=R_{11} / R_{9}
$$

We have chosen the YM coupling (22)

$$
g_{Y M}^{2}=g_{B}
$$

for this case, so that the limit of strongly coupled SYM corresponds to the limit of free Dstrings. We refer to this interpretation as $\mathcal{B}_{D}$. If we now make the $9 \leftrightarrow 11$ flip, as above, we invert the string coupling,

$$
g_{B}=R_{9} / R_{11}
$$

i.e. we do an S-duality operation, therefore it is natural to think that we end up in this way with a type IIB theory in which fundamental strings replace D-strings and vice versa. The action (17) together with

$$
g_{Y M}^{2}=1 / g_{B}^{2}
$$

now describes $N$ fundamental strings with 'coordinates' $X^{i}, \theta^{i}$. The strongly coupled SYM corresponds to weekly coupled fundamental IIB strings. For this reason we call it $\mathcal{B}_{F}$. Finally it is to be expected that a T-duality operation in the $9^{\text {th }}$ direction will map $\mathcal{B}_{F}$ back to $\mathcal{A}_{F}$. In sum we have the diagram

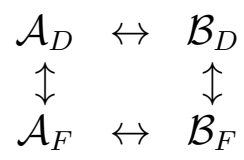

where vertical arrows represent the 9-11 flip, while the horizontal ones represent the T-duality operation. A similar diagram has appeared in [3]. (17) with the specifications (20 23) provides a suitable description for all these cases.

The stringy interpretation of the SYM model (17) is clearer by going to the strong $g_{Y M}$ limit. This limit corresponds to strongly coupled gauge dynamics and is governed by an IR 
fixed point theory. In this limit, the fields $X$ and $\theta$ become diagonal and for this reason, a stringy interpretation is apparent. In (20-23), we have arranged the $g_{Y M}$ so that this limit corresponds to an appropriate string picture in each case. It was argued in [13, 3n that there is a nontrivial identification by the symmetric group $S_{N}$ and the IR theory is the $\mathcal{N}=8$ supersymmetric sigma model conformal field theory

$$
S=\frac{1}{2 \pi} \int \operatorname{tr}\left(\left(\partial_{\mu} X^{i}\right)^{2}+\theta^{T} \not \partial \theta\right)
$$

with the orbifold target space

$$
S^{N} \mathbf{R}^{8}=\left(\mathbf{R}^{8}\right)^{N} / S_{N}
$$

The two spinors $\theta_{L}$ and $\theta_{R}$ have opposite $S O(8)$ chirality, which is the chirality setting for IIA strings in the light cone gauge. This fact was used by [1, 2, 3] to identify the untwisted sector of the orbifold field theory with $N$ IIA strings in the light cone gauge, while twisted sectors give IIA strings of different lengths.

The above interpretations are rather natural; however, as we have already pointed out, when talking about type IIA or IIB, we do not refer simply to the old type IIA and IIB string theories, but to enlarged theories which contain the old string theories as a particular subsector. The rich structure of these theories come directly from the analysis of the SYM model, underlying all of them, which we have done in the last section. In general all four theories split into subsectors labeled by two integers $(m, n)$. They come either from the quantization of the boundary conditions (the cycles of the previous section) or from the quantization of the gauge potential degrees of freedom (the common value of a cycle of $\pi_{k}$ 's which are all identical). In this regard we notice that the energy of the latter (10) is infinite in the stringy limit. It is understood that we are using this limit only to identify the various distinct physical configurations.

Let us first see how the IIA picture emerges in our framework, i.e. from properties of the gauge field moduli space. To this end, we simply go back to the results of the previous section. Consider a sector of the $U(N)$ gauge theory characterized by some definite twisted boundary condition on the gauge fixed configuration (3) for $A_{\mu}$,

$$
A_{\mu}(\sigma+2 \pi)=g A_{\mu}(\sigma) g^{\dagger}
$$

where $g$ is a representative of a non-trivial conjugacy class in $S_{N}$. This induces the following twisted boundary condition on $X_{m n}^{i}$,

$$
X^{i}(\sigma+2 \pi)=g X^{i}(\sigma) g^{\dagger} .
$$

We have already noticed that, since (3) is independent of $\sigma$, (31) implies

$$
\beta_{g(i)}=\beta_{i} .
$$

The physical meaning of this sector is evident in the free string limit, (32) gives

$$
X_{g(i)}(\sigma+2 \pi)=X_{i}(\sigma)
$$


These are long string configurations. To be more specific, consider a particular sector of the gauge theory specified by the conjugacy class

$$
[g]=(1)^{N_{1}}(2)^{N_{2}} \cdots(s)^{N_{s}}
$$

We are going to have

$$
\pi_{m_{1}}^{i}=\pi_{m_{2}}^{i}=\cdots \pi_{m_{k}}^{i}, \quad k=1, \cdots, s, \quad i=1, \cdots, N_{k}
$$

which gives rise to a set of $N_{k}$ strings of length $2 \pi k, k=1, \cdots, s$. Intuitively, we can think of the process of 'screwing' strings as follows. A generic point in the gauge theory moduli space has $\pi_{k}$ all different. This corresponds to $N$ strings with different electric flux circulating the strings. Due to conservation of electric flux, two strings with different flux cannot join. However, corresponding to the point (36) in the moduli space for which a number of strings have the same flux living on them, they can combine with each other and give rise to long strings.

What we have said so far about the $\mathcal{A}_{F}$ theory is not complete. The 'quantum moduli space' of $\mathrm{SYM}_{1+1}$ is characterized by another quantum number, the common value of the $\pi_{k}$ in a given cycle, which has already appeared in (36) above without interpretation. We recall that the trace of the electric flux is usually interpreted as a D0-brane charge, [4, 14, 15]. To see more clearly the relation of theses D0-branes with the long strings, it is convenient to switch to a IIB picture. So we do a T-duality on $\mathcal{A}_{D}$ and land on $\mathcal{B}_{D}$. In a IIB picture we expect D-strings, elementary strings and bound states of them, with integral charges $\left(q_{e}, q_{m}\right)$. We show now the SYM gauge moduli space has a natural interpretation in terms of these charges. To see this, it is enough to look at a particular subsector of the gauge theory, for example, a cycle of length $n$ with an 'electric flux',

$$
\pi_{1}=\pi_{2}=\cdots=\pi_{n}=m, \quad m \geq 0 .
$$

This corresponds to a long string of length $2 \pi n$ in the $\mathcal{A}_{F}$ picture, and according to T-duality, should correspond to a certain D-string configuration. Indeed a long string of length $2 \pi n$ can be thought of as coming from $n$ fundamental IIA strings. One can do a combined T-duality and $\mathrm{S}$-duality operation. It is expected that this long string be mapped to $n$ D-strings in the $\mathcal{B}_{D}$ picture. One can also see this more intuitively by rescaling the long string back to standard world sheet dimension. Since the total mass of the string is not changed, the tension will get increased by a factor of $n$. This can be identified with the tension of a D-string carrying RR-charge $n$ [16],

$$
T \sim n / g_{B}
$$

Next we identify (37) with a IIB string of type $(m, n)$. One can compute the energy per unit length associated with (37),

$$
\mathcal{E} / L=\frac{g_{Y M}^{2}}{2 n} m^{2}
$$


This is, to lowest order, exactly what one would expect $m$ fundamental IIB strings to contribute to the IIB $(m, n)$ string tension,

$$
T_{(m, n)}=\sqrt{m^{2}+\frac{n^{2}}{g_{B}^{2}}} \sim \frac{n}{g_{B}}+\frac{m^{2}}{2 n} g_{B} .
$$

Therefore a long string of length $2 \pi n$, together with the electric flux (37) can be mapped to a collection of $n$ D-strings together with $m$ fundamental strings, i.e. a IIB $(m, n)$ string according to 17 .

Thus one can take the two dimensional $U(N)$ SYM (17) as describing a collection of $(m, n)$ IIB strings, with $n \leq N$, stretched in the $9^{t h}$ direction of radius $1 / R_{9}$. The large $N$ limit will restore the full spectrum required by the $S L(2, \mathbf{Z})$ symmetry of IIB string theory. Stability of $(m, n)$ strings requires $m, n$ to be relatively prime, but this cannot be seen solely from the properties of pure gauge sector and is related to the detailed properties of the full supersymmetric system, [17].

Let us now switch back to $\mathcal{A}_{F}$. Since fundamental strings in $\mathcal{B}_{D}$ are mapped to D0-branes in $\mathcal{A}_{F}$, we see that an $(m, n)$ sector of SYM corresponds to $m$ D0-branes attached to a long string of length $2 n \pi$. This is of course consistent. (37) gives an electric flux

$$
\frac{1}{g_{Y M}^{2}} \operatorname{tr} E=\frac{1}{n} \sum_{k} \pi_{k}=m
$$

of $m$ units and therefore represents $m$ D0 branes. The energy $\mathcal{E}=\frac{g_{Y M}^{2}}{2 n} \mathrm{Lm}^{2}$ is also what one would expect [3] from a configuration of $m$ D0 branes adhering to the long string.

Let us now comment on the inclusion of all the twisted sectors in the IIA string interpretation [1, 2, 3]. It is common in string orbifold construction to include twisted sectors in the orbifold Hilbert space. Precisely which twisted sectors have to be included depends on the physical problem. For ordinary string orbifolds, modular invariance requires the inclusion of all the twisted sectors. Now, let us go back to our SYM. We just saw that the 'long strings configurations' can be identified with IIB $(m, n)$ strings. It is clear that from the requirement of $S L(2, \mathbf{Z})$ symmetry of type IIB theory, we should include all of them, i.e. in $\mathcal{B}_{D}$ we should include all the $(m, n)$ subsectors. This also entails that the same sectors should be present on the IIA side. In other words the $S L(2, \mathbf{Z})$ symmetry of type IIB theory plays, in $\mathrm{SYM}_{1+1}$ of $\mathrm{M}$ (atrix) theory compactifications, a role analogous to modular invariance in ordinary string orbifolds .

Finally, we comment briefly on $\mathcal{B}_{F}$. We pass from $\mathcal{B}_{D}$ to it via a 9-11 flip, which corresponds to an S-duality operation. Therefore D-strings are mapped to fundamental strings and vice versa and we have the opposite assignment of the quantum numbers $n$ and $m$, with respect to above. In other words, the original IIA long strings of $\mathcal{A}_{F}$ have become now the fundamental strings in $\mathcal{B}_{F}$ and are assigned the quantum number $n$. This is of course consistent, since the relation between $\mathcal{B}_{F}$ and $\mathcal{A}_{F}$ is a T-duality operation, which leaves unchanged the $\mathrm{D}$ - or $\mathrm{F}$-character of the string configurations. In fact, (39) in this case becomes $\frac{m^{2}}{2 n g_{B}^{2}}$, which agrees 
with what one would expect from the tension of a $(n, m)$ string,

$$
T_{(n, m)}=\sqrt{n^{2}+\frac{m^{2}}{g_{B}^{2}}} \sim n+\frac{m^{2}}{2 n g_{B}^{2}} .
$$

\section{Discussions}

Throughout this paper we have kept $N, R_{9}$ and $R_{11}$ finite. We have interpreted SYM as an approximate description of 9-dimensional theories, in particular (17) with (22) as an effective description of D-strings. This theory was originally used by Witten, [17], as an effective description of coincident IIB D-strings to study bound state problems. Given the M/IIB duality (see for example [18]), one can think of it as a partial description of $\mathrm{M}$ theory compactified on a torus with radii $R_{9}, R_{11}$.

We have seen above that we can recover the full IIB (and consequently also the IIA) spectrum by taking $N \rightarrow \infty$. Therefore a discussion of this limit is unavoidable. The best we can say is that in the framework of $\mathrm{M}$ (atrix) theory, the issue of large $N$ limit seems far from being well established. On the one hand we can take the original attitude of [4] and say that (16) in the $N \rightarrow \infty$ and $R_{11} \rightarrow \infty$ limit represents 11-dimensional $\mathrm{M}$ theory. On the other hand, we can think of taking the $N \rightarrow \infty$ limit, while keeping $R_{11}$ finite and ask ourselves whether this corresponds to any sensible theory (in 10D). We do not have a decisive argument to choose this second attitude. However, if we consider further compactification on a circle of radius $R_{9}$, as in the last section, given the nice interpretations of SYM presented there, which agree very well with the notions we have about type IIA and type IIB theories in 9-dimensions, we are oriented to assume that the $N \rightarrow \infty$ limits of the theories (17) represent enlarged IIA and IIB theories in 9 dimensions. This seems somehow to imply that (16) represents in the large $N$ limit $\mathrm{M}$ theory compactified on a circle.

Assuming this, (17), which was an effective description for 9D theory, becomes in the large $N$ limit an exact description of $\mathrm{M}$ theory compactified on a torus. Thus, the two dimensional SYM provides a unified description of both IIA and IIB. Let us comment about this possibility. According to [18], the $\mathrm{M}$ theory membrane can wrap on a torus in different ways and give rise to the various IIB $(m, n)$ strings. To recover 10 dimensional IIB, one should let $R_{9}, R_{11} \rightarrow 0$, with $g_{B}=R_{11} / R_{9}$ fixed. The choice (22) of $g_{Y M}$ in (17) is appropriate for this case. On the other hand we can take $R_{11} \rightarrow \infty$ and consider M theory compactified on a circle, which must coincide with IIA. This corresponds to the choice (21) made in [3], which is suitable for this purpose, since $R_{11}$ does not appear in (18).

Acknowledgements. We would like to acknowledge useful discussions we had with PeiMing Ho and Cesare Reina. This research was partially supported by EC TMR Programme, grant FMRX-CT96-0012.

Note added: While this work was being typed, the paper [19] which also propose to treat the two dimensional SYM as describing IIB fundamental strings. 


\section{Appendix}

In this appendix, we repeat the procedure of [11], We start with the D0 brane action (16) and rescale $X^{i}$ so that

$$
S=\int d t L=\int d t \frac{1}{2 R_{11}^{3}} \operatorname{tr}\left(\dot{X}^{i 2}-\left[X^{i}, X^{j}\right]^{2}+\theta^{T} \dot{\theta}-\theta^{T} \gamma_{i}\left[X^{i}, \theta\right]\right)
$$

Compactifing this on a circle of radius $R_{9}$, we obtain (without explicitly writing the fermionic terms)

$$
S=\int d t L=\int d t \int_{0}^{2 \pi / R_{9}} d \sigma \frac{R_{9}}{2 R_{11}^{3}} \operatorname{tr}\left(\dot{X}^{i 2}+\dot{A}_{1}^{2}-\left(D_{\sigma} X^{i}\right)^{2}-\left[X^{i}, X^{j}\right]^{2}\right) .
$$

Rescaling the world sheet back to normal length $2 \pi$, and also rescaling $A_{1}$ such that $D_{\sigma} \rightarrow$ $R_{9} D_{\sigma}$

$$
S=\frac{1}{2} \int d t \int_{0}^{2 \pi} d \sigma t r\left(\frac{\dot{X}^{i 2}}{R_{11}^{3}}+\frac{R_{9}^{2}}{R_{11}^{3}} \dot{A}_{1}^{2}-\frac{R_{9}^{2}}{R_{11}^{3}}\left(D_{\sigma} X^{i}\right)^{2}-\frac{1}{R_{11}^{3}}\left[X^{i}, X^{j}\right]^{2} .\right)
$$

Now rescale the coordinates $X \rightarrow\left(R_{11}^{3} / R_{9}\right)^{1 / 2} X$

$$
\begin{aligned}
S & =\frac{1}{2} \int d t \int_{0}^{2 \pi} d \sigma t r\left(\frac{\dot{X}^{i 2}}{R_{9}}+\frac{R_{9}^{2}}{R_{11}^{3}} \dot{A}_{1}^{2}-R_{9}\left(D_{\sigma} X^{i}\right)^{2}-\frac{R_{11}^{3}}{R_{9}^{2}}\left[X^{i}, X^{j}\right]^{2}\right) \\
& :=\int d t \int_{0}^{2 \pi} d \sigma \mathcal{L} .
\end{aligned}
$$

Using $\mathcal{L}$, one can construct the corresponding Hamiltonian

$$
H=\frac{R_{9}}{2} \int_{0}^{2 \pi} d \sigma t r\left(\Pi_{i}^{2}+\left(D_{\sigma} X^{i}\right)^{2}+\left(\frac{R_{11}}{R_{9}}\right)^{3}\left(\Pi_{A}^{2}+\left[X^{i}, X^{j}\right]^{2}\right)\right)
$$

where $\Pi_{A}$ is the conjugate momentum of $A_{1}$. The gauge invariant form of the action can be obtained by absorbing the overall factor of $R_{9}$ into the definition of world sheet time in (46): $t \rightarrow t / R_{9}$

$$
\left.S=\frac{1}{2} \int d t \int_{0}^{2 \pi} d \sigma t r\left(\left(D_{\mu} X^{i}\right)^{2}+\frac{R_{9}^{3}}{R_{11}^{3}} F_{\mu \nu}^{2}-\frac{R_{11}^{3}}{R_{9}^{3}}\left[X^{i}, X^{j}\right]^{2}\right) .\right)
$$

Different dependences of the Yang-Mills couplings, such as those of eqs.(20 23), can be obtained by noticing that the explicit dependence of $R_{11}$ in (43) can be removed or, anyhow, modified by means of field redefinitions together with a redefinition of time.

We believe world-sheet time redefinitions are not irrelevant in defining the appropriate large $N$ limit. 


\section{References}

[1] L. Motl, Proposals on Nonperturbative Superstring Interactions, hep-th/9701025.

[2] T. Banks and N. Seiberg, Strings from Matrices, hep-th/9702187.

[3] R. Dijkgraaf, E. Verlinde, H. Verlinde, Matrix String Theory, hep-th/9703030.

[4] T. Banks, W. Fischler, S. H. Shenker and L. Susskind, M Theory As A Matrix Model: A Conjecture, hep-th/9610043.

[5] R. Dijkgraaf, G, Moore, E. Verlinde, H. Verlinde, Elliptic Genera of Symmetric Products and Second Quantized Strings, Commun. Math. Phys. to be published, hep-th/9608096.

[6] J.E.Hetrick and Y.Hosotani Yang-Mills Theory on a Circle, Phys.Lett. B230 (1989), 88.

[7] E.Langmann and G.W.Semenoff, Gauge Theory on a Cylinder, Phys.Lett. B296 (1992), 117, hep-th/9210011. Gribov Ambiguity and Non-Trivial Vacuum Structure of Gauge Theories on a Cylinder, Phys.Lett. B303 (1993), 303,hep-th/9212083.

[8] J.E.Hetrick, Canonical Quantization of Two Dimensional Gauge Fields, Int. Jour. Mod. Phys. A9 (1994), 3153, hep-th/9305020.

[9] S.G.Rajeev, Yang-Mills Theory on a Cylinder, Phys.Lett. B212 (1988), 203.

[10] U.H. Danielsson, G. Ferretti, and B. Sundborg, D-particle Dynamics and Bound States, Int. J. Mod. Phys. A11 (1996) 5463-5478, hep-th/9603081;

D. Kabat and P. Pouliot, A Comment on Zerobrane Quantum Mechanics, Phys. Rev. Lett. 77 (1996) 1004-1007, hep-th/9603127;

M. R. Douglas, D. Kabat, P. Pouliot, and S. H. Shenker, D-branes and Short Distances in String Theory, Nucl. Phys. B485 (1997) 85-127, hep-th/9608024.

[11] W. Taylor, D-brane Field Theory on Compact Spaces, hep-th/9611042.

[12] P.M. Ho and Y..S. Wu, IIB/M Duality and Longitudinal Membranes, hep-th/9703016.

[13] J.A. Harvey, G. Moore, and A. Strominger, Reducing S-Duality to T-Duality, Phys.Rev. D52 (1995) 7161, hep-th/9501022.

M. Bershadsky, A. Johansen, V. Sadov, C. Vafa, Topological Reduction of $4 D$ SYM to $2 D$ $\sigma-M o d e l$, Nucl. Phys. B448 (1995) 166, hep-th/9501096.

[14] O. J. Ganor, S. Ramgoolam and W. Taylor, Branes, Fluxes and Duality in M(atrix)Theory, hep-th/9611202.

[15] T.Banks, N.Seiberg and S.Shenker, Branes from Matrices, hep-th/9612157.

[16] J. Polchinski, Dirichlet-Branes and Ramond-Ramond Charges, hep-th/951001. 
[17] E. Witten, Bound States of Strings and p-Branes, Nucl. Phys. B460 (1996) 335, hep-th/9510135.

[18] J. H. Schwarz, Lectures on Superstring and M Theory Dualities, hep-th/9607201.

[19] H.Verlinde, A Matrix String Interpretation of the Large $N$ Loop Equation, hep-th/9705029. 